\title{
QUEEN'S
UNIVERSITY
BELFAST
}

\section{Sputum trypsin-like protease activity relates to clinical outcome in cystic fibrosis}

Reihill, J., Moffitt, K., Douglas, L., Elborn, S., Jones, A., \& Martin, L. (2020). Sputum trypsin-like protease activity relates to clinical outcome in cystic fibrosis. Journal of Cystic Fibrosis. https://doi.org/10.1016/j.jcf.2019.12.014

\author{
Published in: \\ Journal of Cystic Fibrosis
}

\section{Document Version:}

Peer reviewed version

Queen's University Belfast - Research Portal:

Link to publication record in Queen's University Belfast Research Portal

\section{Publisher rights}

Copyright 2020 Elsevier.

This manuscript is distributed under a Creative Commons Attribution-NonCommercial-NoDerivs License

(https://creativecommons.org/licenses/by-nc-nd/4.0/), which permits distribution and reproduction for non-commercial purposes, provided the author and source are cited.

\section{General rights}

Copyright for the publications made accessible via the Queen's University Belfast Research Portal is retained by the author(s) and / or other copyright owners and it is a condition of accessing these publications that users recognise and abide by the legal requirements associated with these rights.

\section{Take down policy}

The Research Portal is Queen's institutional repository that provides access to Queen's research output. Every effort has been made to ensure that content in the Research Portal does not infringe any person's rights, or applicable UK laws. If you discover content in the Research Portal that you believe breaches copyright or violates any law, please contact openaccess@qub.ac.uk. 


\title{
Sputum trypsin-like protease activity relates to clinical outcome in cystic fibrosis
}

James A. Reihill ${ }^{1}$, Kelly L. Moffitt ${ }^{1}$, Lisa E.J. Douglas ${ }^{1}$, J. Stuart Elborn ${ }^{2}$, Andrew M. Jones ${ }^{3}$

$\& *$ S. Lorraine Martin ${ }^{1}$

${ }^{1}$ School of Pharmacy, ${ }^{2}$ Wellcome-Wolfson Institute For Experimental Medicine, Queen's University Belfast, Northern Ireland, BT9 7BL, UK.

${ }^{3}$ Wythenshawe Hospital, Manchester Adult Cystic Fibrosis Centre, South Moor Road, Manchester, M23 9LT, UK.

*Corresponding author

Email:1.martin@qub.ac.uk

Telephone: +44 (0)28 90975711

\begin{abstract}
Background: In cystic fibrosis (CF) airways excessive levels of serine trypsin-like proteases (TLP) activate the epithelial sodium channel $(\mathrm{ENaC})$ resulting in airways dehydration and promotion of mucus secretion. Despite this the relationship of TL proteolytic activity and clinical outcome has not been studied.
\end{abstract}

Methods: We analysed supernatant (sol) prepared from CF sputum from 29 and 33 adult CF patients in two study cohorts, respectively. Protease activities were determined by measuring the hydrolysis of peptide-based substrates or by ELISA. Lung function was assessed by spirometry $\left(\mathrm{FEV}_{1}\right)$. Mortality data was retrospectively obtained and time in months until death or transplantation used for subsequent survival analysis.

Results: TLP activity inversely correlated with percent predicted $\mathrm{FEV}_{1}(\mathrm{r}=-0.4, \mathrm{p}=0.03)$ and was greater in individuals who did not survive beyond 5-years from the time of sample collection. A Kaplan-Meier analysis demonstrated significantly reduced survival $(\mathrm{p}=0.04)$ for individuals with high TLP activity [hazard ratio (HR) of 7.21 (per log unit TLP activity $(\mathrm{p}=0.03)]$. In contrast, neutrophil elastase displayed no significant associations with lung function or patient survival. Similar findings were evident in a second study cohort.

Conclusions: Sputum TLP activity may represent a novel non-invasive biomarker and/or therapeutic target for CF lung disease.

Keywords: Cystic fibrosis; trypsin-like proteases; lung function; patient survival; $\mathrm{ENaC}$ 


\section{Introduction}

Sputum biomarkers hold promise as a non-invasive means to monitor cystic fibrosis (CF) lung disease. In particular, levels of sputum IL-8 and neutrophil elastase (NE) have been found to have a significant inverse association with lung function decline $(1,2)$, with NE the only biomarker analysed to date, to show a longitudinal association with $\mathrm{FEV}_{1}$ (1). NE however, has not been shown to predict patient survival (3). Rather, the inflammatory cytokine HMGB1 (high-mobility group box-1 protein) is the only biomarker reported to have this association (4). The identification of other candidate markers of potential prognostic value is therefore important as a variance in pulmonary phenotype and survival is evident in patients with identical CFTR mutations (5).

A protease-antiprotease imbalance in $\mathrm{CF}$ airways is well reported with $\mathrm{NE}$ the dominant activity routinely measured (6). In addition, matrix metalloproteases such as MMP-2, -9 and 12 and the cysteine proteases, cathepsin $\mathrm{B}$ and $\mathrm{S}$ have received significant study which has contributed to a dissection of their possible roles in the pathogenesis of CF lung disease (7, 8). In contrast, there is a dearth of reports investigating trypsin-like protease (TLP) activity in clinical samples even through this sub-group of serine proteases (both membrane-bound and soluble) have the potential to contribute to a number of disease-relevant processes such as activation of the epithelial sodium channel $(\mathrm{ENaC})$ and the stimulation of a mucus secretory phenotype and inflammatory signalling pathways, potentially through the activation of protease-activated receptor-2 (PAR-2) $(9,10)$.

Dysregulated ion transport is a key feature of CF airway epithelium, where in addition to the loss of CF transmembrane conductance regulator (CFTR) function, concomitant hyperactivity of $\mathrm{ENaC}$ underlies rapid $\mathrm{Na}^{+}$and water absorption from the luminal surface of the conducting airways $(11,12)$. ENaC is composed of three structurally related subunits $(\alpha, \beta$ and $\gamma)$, which include two membrane-spanning domains connected by a large extracellular loop. Although $\mathrm{ENaC}$ can be regulated by multiple pathways e.g. cAMP and SPLUNC-1, it is activated by the proteolytic processing of its subunits (11). Extracellular trypsin is known to increase channel activity and inhibition of TL serine proteases by broad-spectrum, macromolecular protease inhibitors such as aprotinin and camostat and QUB-TL1, both low molecular weight inhibitors, have been shown to attenuate $\mathrm{ENaC}$ and improve mucociliary clearance (13). Prostasin (a typeII transmembrane serine protease) has been identified as a candidate Channel Activating 
Protease (CAP-1). Further efforts have been made to identify other potential CAPs and have determined multiple Arg and Lys cleavage sites e.g. $\gamma \operatorname{Lys}^{194}$ (plasmin); $\alpha \operatorname{Arg}^{205}, \alpha \operatorname{Arg}^{231}$ and $\gamma \operatorname{Arg}^{143}$ (furin); $\gamma \operatorname{Lys}^{186}$ (prostasin) and $\gamma \operatorname{Arg}^{138}$ (CAP-2; TMPRSS4) (14). In CF airways excessive TLP activity to include putative prostasin and matriptase activities, promotes ENaCmediated fluid absorption $(9,15,16)$, with the resultant dehydration of the airway surface liquid (ASL) layer regarded as a key initiating event for disease pathogenesis (17).

PARs are $G$ protein-coupled receptors which are activated by the proteolytic cleavage of a ligand on the extracellular N-terminus. There are four PARs; thrombin activates PAR-1, -3, and -4 whereas TL proteases activate PAR-2 and -4. PAR-2 is expressed basolaterally in human bronchial epithelial cells at air-liquid interface where it stimulates intracellular $\mathrm{Ca}^{2+}$ release and $\mathrm{Ca}^{2+}$ influx, resulting in a concomitant activation of low-level nitric oxide production and an increase in apical membrane $\mathrm{Cl}^{-}$permeability $(\sim 3.5$ fold $)$ with increases in ciliary beating observed by up to $50 \%$ (10). Other studies have shown PAR-2 regulation of airway cell cytokine and mucus secretion. Pathogens (bacterial and fungal) also secrete proteases that activate PARs as can a number of allergen-associated proteases (18). PAR-2 may however, be disarmed by some elastases and cathepsin G (19) via extracellular domain cleavage downstream of the activating site, preventing trypsin activation but not activation by peptide agonists (20). In addition to the impact on ENaC and PAR-2 pathways, TLP activity, such as human airways trypsin-like protease (HAT) enhance mucin (MUC2 and MUC5A) gene expression and stimulate mucus hypersecretion through a amphiregulin (AR)-EGFR pathway $(21,22)$. Host TLPs such as TMPRSS2, TMPRSS4, HAT, matriptase, kallikreins and tryptase are also implicated in the activation of a large number of viruses such as influenza (23).

Together these in vitro studies suggest TLPs, a previously overlooked subset of the serine protease superfamily, may play an important pathophysiological role in the CF lung. Although we and others have highlighted previously strategies to regulate $\mathrm{ENaC}$ through the targeting of associated TL channel activating proteases, previous protease biomarker studies have focused on neutrophil-derived enzymes such as NE with no reports to date investigating epithelial derived TLPs as putative markers of disease severity or progression. As such the aim of this study was to determine whether there is any association between TLP activity measured in CF sputum sol and lung function and patient outcome. 


\section{Materials and methods}

\subsection{Study cohorts}

Study cohort 1 was previously reported in an examination of inflammatory markers in stable CF patients with transmissible and sporadic strains of Pseudomonas aeruginosa with recruitment of patients and study design reported in detail (24). A second cohort (study cohort 2) consists of 33 adult CF patients recruited at the Belfast City Hospital (during exacerbation) between March-September 2009. All adult inpatients with the ability to provide a sputum sample were included in the study except those with active mycobacterium infection or haemoptysis. The following baseline variables were recorded at inclusion: age, gender, BMI and lung function ( $\mathrm{FEV}_{1} ; \%$ predicted). Pseudomonas aeruginosa was identified in $70 \%$ of the cohort (23 out of 33 patients). For both study cohorts mortality data was retrospectively obtained and the time in months until death or transplantation used for survival analysis.

\subsection{Sputum induction}

Expectorated sputum samples were obtained from patients, and diluted with $0.9 \%(\mathrm{w} / \mathrm{v})$ saline in a ratio of 1:5 and the sample vortexed for 30 seconds. A centrifugation step (3,000 $\mathrm{g}$ for 30 minutes at $4^{\circ} \mathrm{C}$ ) facilitated the collection of an aqueous supernatant (sol), which was aliquoted and frozen at $-80^{\circ} \mathrm{C}$ for subsequent analysis. Dithiothreitol (DTT) was not included at any time during processing.

\subsection{Biochemical measurements}

TLP activity was assayed in sputum sol (25 $\mu$ l in assay buffer (PBS); final volume $100 \mu \mathrm{l}$ ) by monitoring the hydrolysis of $50 \mu \mathrm{M}$ Boc-QAR-NH${ }_{2} \mathrm{Mec}$ (R\&D systems) with assay buffer used as a vehicle control. The rate of substrate hydrolysis was continuously recorded using $\lambda_{\text {ex }} 360$ $\mathrm{nm}$ and $\lambda_{\mathrm{em}} 480 \mathrm{~nm}$, over a period of 60 minutes (FLUOSTAR Optima microplate reader; BMG Labtech). In a complex biological material such as sputum sol it is important to mitigate against non-specific hydrolysis of a peptide-based substrate by host- and pathogen-derived enzymes from alternate protease classes (e.g. metalloproteinase or cysteine). As such, sputum samples were treated in the presence or absence of $10 \mu \mathrm{M}$ QUB-TL1 which enabled determination of the QUB-TL1 sensitive portion (where the inhibited result was subtracted from the uninhibited activity data). QUB-TL1 was utilised for this purpose as it is a broad spectrum, irreversible inhibitor of serine trypsin-like proteases (9). Importantly, this compound does not inhibit activities from non-trypsin-like serine proteases such as neutrophil elastase and chymotrypsin 
nor does it inhibit alternate proteases species relevant to the CF lung environment (Supplemental Figure S1). Active NE was measured using the ProteaseTag ${ }^{\circledR}$ Active NE Immunoassay kit (ProAxsis Ltd.) (25). Protease activity measurements were performed in duplicate with CV values $<10 \%$ deemed acceptable.

\subsection{Statistical analysis}

Descriptive statistics were used to describe the study cohorts at baseline. Throughout the study normality was determined using the Shapiro-Wilk test. For the comparison of study cohort 1 with study cohort 2 categoric variables were summarised as frequencies and comparisons performed by Fishers exact test. Comparisons between continuous variables were by two-tailed Mann-Whitney analysis. Data are expressed as mean (SD) for normally distributed values or median (IQR) for non-Gaussian distributed data. Spearman's rank correlation coefficient was used to measure the strength of the linear association between protease activities and inflammatory biomarkers/clinical parameters. Patient survival was first estimated using Kaplan-Meier survival analysis with the patient cohort separated into two groups based on the median protease activity value. The Gehan-Breslow-Wilcoxon test was used for statistical analysis. The relationship between protease activities and patient survival was determined using Cox proportional hazard modelling which calculated hazard ratios (expressed per log unit protease activity) and 95\% confidence intervals. Adjustments for individual level confounders (such as age, gender, BMI) were on the basis on associations $(p<0.10)$ on univariate analysis. Statistical analysis was performed using the SPSS (SPSS version 22, Chicago, Illinois, USA) software package.

\subsection{Study approval}

Approval for the study was obtained from the South Manchester Research Ethics Committee or the Health and Social Care Research Ethics Committee Northern Ireland as appropriate. Written informed consent was obtained from all patients at the time of recruitment. Experiments were performed in accordance with relevant approved guidelines and regulations. 


\section{Results}

\subsection{Description of the study cohorts}

The baseline characteristics of the study cohorts are outlined in Table 1. Study cohort 1 consisted of 29 clinically stable adult subjects ( $>18$ years old) with a median age of 26.3 (IQR 20.5-32.5). Females comprised $62 \%$ of the group. The baseline median percent predicted $\mathrm{FEV}_{1}$ (ppFEV $)_{1}$ was 41\% (IQR 32.3-59.3). Subjects had a median (IQR) body mass index (BMI) of $19.9(18.9-21.5) \mathrm{kg} / \mathrm{m}^{2}$. An event (transplant or death) occurred in $17 \%$ of the cohort within 5 years and in $65 \%$ by the end of the study period ( 221 months). Study cohort 2 consisted of 33 adult subjects (hospitalised during exacerbation) with a median age of 25 (IQR 21-33.5) years. Females comprised $52 \%$ of the group. Median ppFEV 1 was $43 \%$ (IQR 29-70) with a median BMI of 20.2 (IQR 18.3-21.6) kg/m². An event (transplant or death) occurred in $33 \%$ of the cohort within 5 years and in $36 \%$ by the end of the study period ( 79 months). We observed no statistically significant differences for each parameter listed in Table 1 when comparing study cohorts 1 and 2; except for comparisons of survivors and non-survivors at the end of the study period ( $\mathrm{p}=0.04$ ). This likely reflects the length of study durations (study cohort 1, 221 months; study cohort 2, 79 months. Indeed, no statistical difference was observed upon analysis of the 5-year mortality data.

Table 1: Description of the baseline characteristics of the study cohorts and patient outcome. Data are displayed either as the number (percent), median (IQR) or mean (SEM). The number of patients alive or dead/transplant is reported for 5 years post sample collection and at the end of the study period.

\begin{tabular}{ccc}
\hline Parameter & Study cohort 1 & Study cohort 2 \\
\hline Population total & 29 & 33 \\
Gender (\% female) & 62 & 52 \\
Age (years) & $26.3(20.5-32.5)$ & $25(21-33.5)$ \\
FEV1 (\% predicted) & $41(32.3-59.3)$ & $43(29-70)$ \\
BMI & $19.9(18.9-21.5)$ & $20.2(18.3-21.6)$ \\
\hline Genotype & & \\
Phe508del/Phe508del & $18(62 \%)$ & $18(55 \%)$ \\
Phe508del/Other & $11(38 \%)$ & $14(42 \%)$ \\
Unknown & - & $1(3 \%)$ \\
Mortality data (5 years) & & \\
Alive & $24(83 \%)$ & $22(67 \%)$ \\
Number of deaths & $5(17 \%)$ & $11(33 \%)$ \\
Number of transplants & 0 & 0 \\
\hline Mortality data (end of study) & & $21(64 \%)$ \\
Alive & $10(35 \%)$ & $11(33 \%)$ \\
Number of deaths & $16(55 \%)$ & $1(3 \%)$ \\
Number of transplants & $3(10 \%)$ & \\
\hline
\end{tabular}




\subsection{TLP activity and lung function}

In study cohort 1 TLP activity inversely correlated with lung function ( $\left.\mathrm{ppFEV}_{1}\right)(\mathrm{r}=-0.4$, $\mathrm{p}=0.03)$ (Figure 1A). A similar trend was observed in study cohort $2(\mathrm{r}=-0.3)$ although this was not statistically significant $(\mathrm{p}=0.1)$ (Figure $1 \mathrm{~B})$.

A

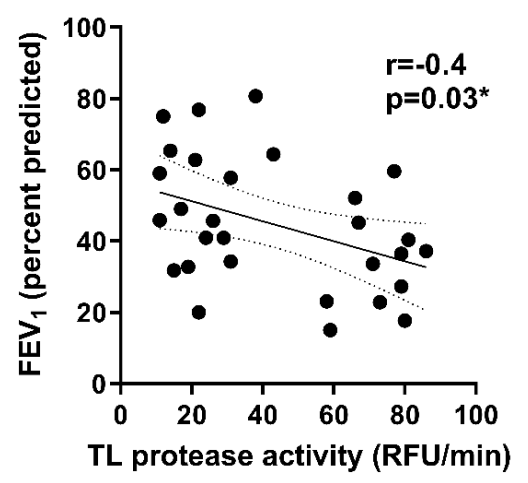

B

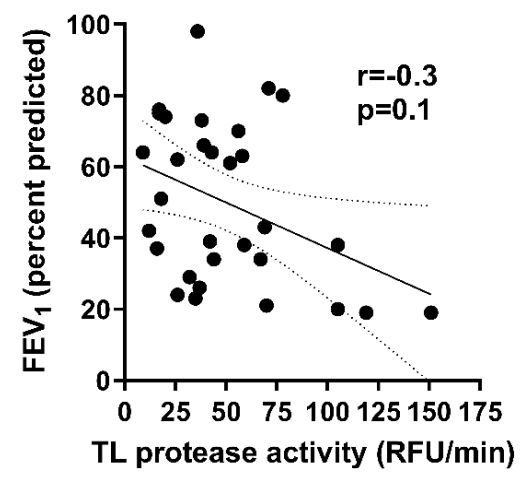

Figure. 1: Correlation analysis for TLP activity and $\mathrm{ppFEV}_{1}$ for (A) study cohort 1 and (B) study cohort 2. Statistical data are presented as Spearman's rank $r$ value ( $p$-value).

\subsection{TLP activity levels and 5-year patient survival}

Study cohort 1: We examined the relationship between sputum TLP activity levels and 5-year survival. Median TLP activity was 2.8 -fold greater in non-survivors compared to surviving counterparts 77 (IQR 58.5-79.5) vs 27.5 (IQR 17.5-66.75) RFU/min; $\mathrm{p}=0.02$ ) (Figure 2A).

Study cohort 2: A 1.5-fold greater level of TLP activity was evident in individuals who did not survive beyond 5 years (60 (IQR 35-105) vs 40.5 (IQR 19.5-58.25) RFU/min for non-survivors and survivors respectively) though this was not statistically significant $(\mathrm{p}=0.1)$ (Figure $2 \mathrm{~B})$.

A

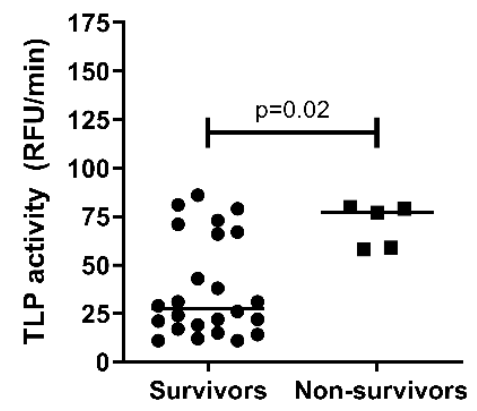

B

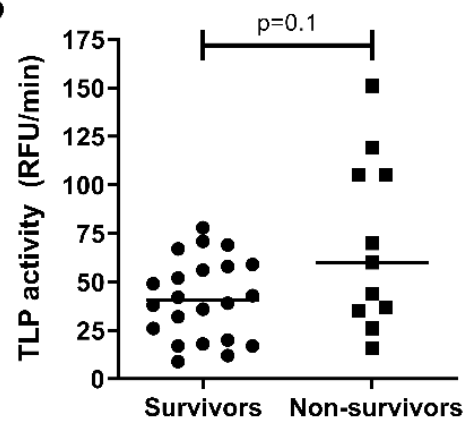

Figure 2: The relationship between sputum TLP activity levels and 5-year patient survival for (A) study cohort 1 and (B) study cohort 2. The median TLP activity value for each group is highlighted by a horizontal bar. Differences between groups was determined using the Mann-Whitney statistical test. 


\subsection{Increased TLP activity levels is associated with poorer patient survival}

Study cohort 1: Kaplan-Meier survival analysis demonstrated individuals with high (above median) TLP activity had a poorer survival outcome compared with individuals with low (below median) TLP activity $(\mathrm{p}=0.04)$ (Figure 3A); median survival time 111 months compared to 204 months (Figure 3A). Increasing TLP activity related to a significant mortality hazard $(\log$ HR 7.21; 95\% CI 1.25-41.6; $p=0.03)$.

Study cohort 2: Initial crude survival analysis (Kaplan-Meier) demonstrated individuals with high TLP activity had a tendency for reduced survival though this was not statistically significant $(\mathrm{p}=0.08)$ (Figure $3 \mathrm{~B})$. Multivariate Cox analysis however, revealed a significant mortality hazard $(\log$ HR 19.2, 95\% CI 1.82-202.7; $\mathrm{p}=0.01)$ with increasing TLP activity.

A

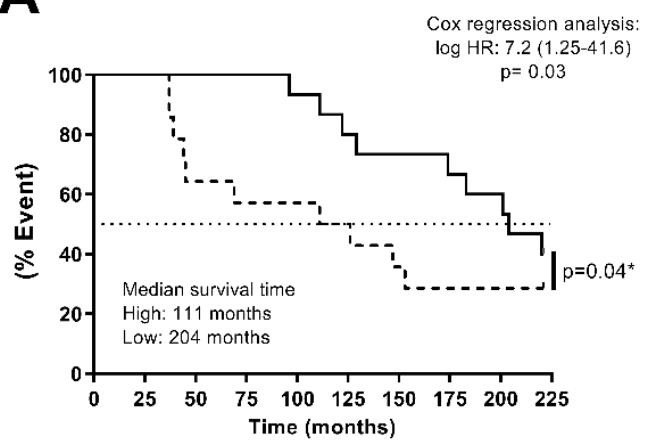

B

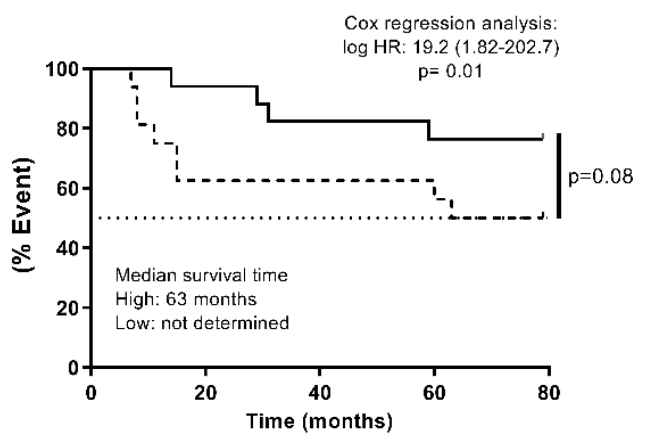

- Below Median

-ᄂ. Above Median

Figure 3: Kaplan-Meier curves for the time to event demonstrating the difference in time to death or censoring (transplantation) for patients with TLP activities above (referred to as high) or below (referred to as low) the median protease activity value for study cohort 1 (A) and study cohort 2 (B). The log hazard ratio was determined separately by Cox regression analysis.

\subsection{TLP activity and treatment}

At the time of sample collection, no patients in cohorts 1 or 2 were on CFTR modulators. A sub-analysis of clinically stable patients (cohort 1) on therapies such as inhaled or oral antibiotics, inhaled or oral steroid and macrolide showed no significant differences in the levels of TLP activity across groups (Supplemental Figure S2); all patients in cohort 2 (exacerbation group) were on various mixtures of inhaled, i.v. and oral antibiotics which limited further analysis. 


\subsection{Comparison of TLP activity with NE}

$\mathrm{NE}$ is well-established as the dominant protease activity associated with CF lung disease pathogenesis. A correlation analysis between NE and TLP activity found no relationship in either study cohort (study cohort $1: \mathrm{r}=0.19, \mathrm{p}=0.3$; study cohort 2 : $\mathrm{r}=0.12, \mathrm{p}=0.5$ ); indicating that these enzymes act in a distinct manner. In contrast to our findings that TLP activity levels related to poorer lung function and patient survival (Figures 1-3) we did not find any relationship between NE levels and lung function (Figure $4 \mathrm{~A} \& \mathrm{~B}$ ), nor with patient survival (Figure 4C\&D), in study cohorts 1 or 2.

A

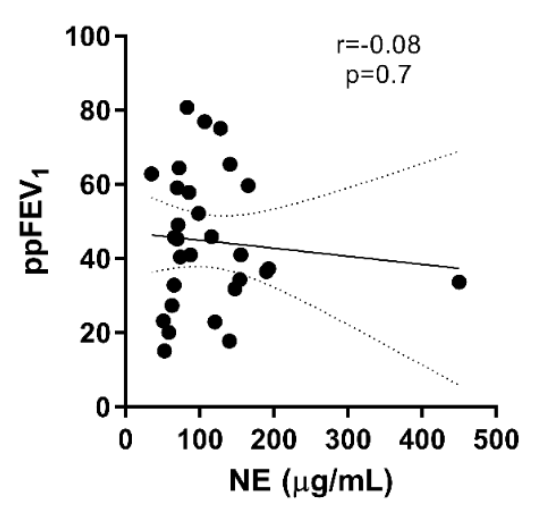

C

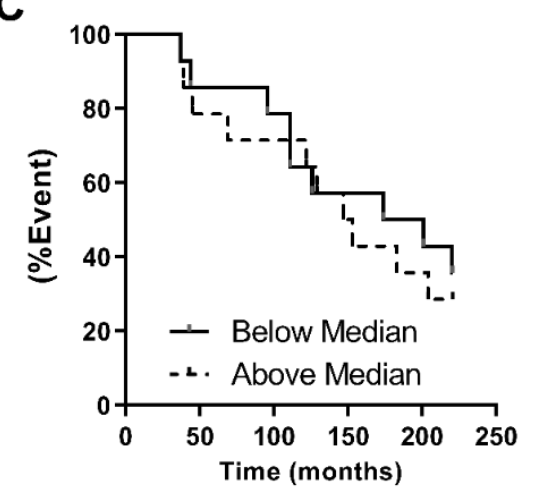

B

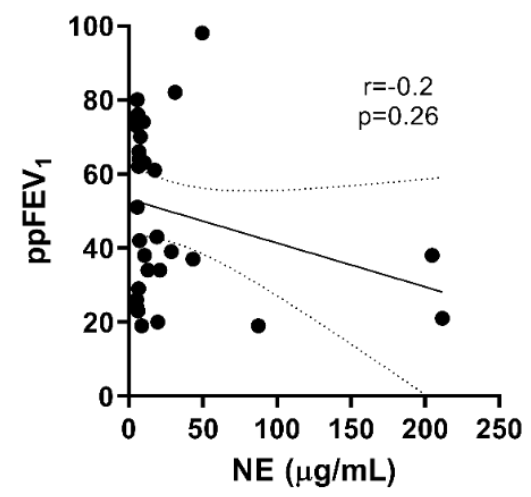

D

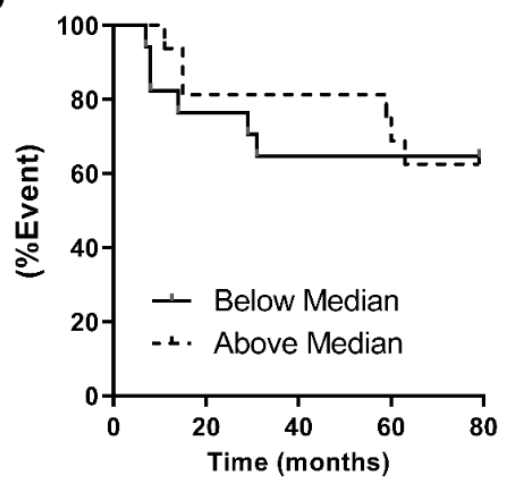

Figure 4. Correlation analysis regarding NE activity and ppFEV ${ }_{1}$ for (A) study cohort 1 and (B) study cohort 2. Statistical data are presented as Spearman's rank r value (p-value) and Kaplan-Meier curves for the time to event demonstrating the difference in time to death or censoring (transplantation) for patients with NE levels above or below the median protease activity value for study cohort 1 (C) and study cohort 2 (D). 


\section{Discussion}

The primary focus of this investigation was an examination of the relationship between TLP activity levels present in adult CF airways (sputum) and clinical parameters of CF lung disease. We report for the first time, that patients with high levels of TLP activity associate with poorer lung function and survival. This is important as the identification of biomarkers predictive of future outcomes may aid our understanding of mechanisms of disease and may assist with the assessment of therapeutic interventions.

A number of secreted, soluble epithelial-derived TLPs are present in CF airways, of which prostasin and matriptase are of particular interest due to reported increased activity levels of these TLPs in CF airway epithelial cells $(9,15,16)$. These activities have the potential to contribute to the hyperactivation of $\mathrm{ENaC}$ and subsequent airways dehydration; a key initiating factor for the development of CF lung disease (17). In addition, human airway trypsin-like protease (HAT) enhances mucin gene expression and mucus hypersecretion $(21,22)$. Inflammatory signalling via PAR-2, leading to inflammatory mediator release and possible leukocyte recruitment, is predominantly activated by trypsin, which is secreted by epithelial cells in addition to nerve cells (20). Feasibly TLP-mediated ENaC activation, inflammation and/or mucus secretion may underlie the associations with lung function and patient survival reported herein.

$\mathrm{NE}$, which has also been shown to activate $\mathrm{ENaC}$ (26) and promote mucus secretion (7), did not associate with lung function decline nor patient mortality further highlighting the complex mechanisms at play in CF airways. NE is secreted in substantial quantities as part of the inflammatory response to the recurrent bacterial infections associated with CF airways disease, contributes to lung damage and has been reported as a risk factor for the onset and progression of lung disease in children with CF (2). Conversely, NE is a fundamental component of innate immunity, with NE-/- mice more susceptible to death upon exposure to Gram-negative infection $(27,28)$. The discordant roles of NE (protective and destructive) in this context may underlie the lack of association between NE and patient survival reported herein. In the Scnn $1 b$ $\mathrm{Tg}$ mouse model of $\mathrm{CF}$ lung disease genetic deletion of $\mathrm{NE}\left(\mathrm{NE}^{-/}\right)$causes reduced inflammation, mucus hypersecretion and structural lung damage but did not reduce airway mucus plugging or pulmonary mortality (29). One previous study examining the relationship between sputum NE levels and CF patient survival did not find any relationship (3). Therefore, 
while NE plays an important role in the progression of CF lung disease it is necessary to consider other additional factors that may contribute to survival.

This current study reports cross-sectional, rather than longitudinal data, which limits our ability to determine causality and furthermore was retrospective in nature. A prospective study, is therefore warranted. Only one other CF sputum biomarker HMGB1 (high-mobility group box1 protein) has been reported as a candidate which predicts CF patient survival. We did attempt to measure HMGB1 by ELISA to allow comparison with our data, however no measurable levels were observed, possibly reflective of reported issues regarding the stability of this protein over long-term storage (30). The lack of association between NE and lung function in our study cohorts was also unexpected although there is a clear disparity between the cohort sizes used in this study and the large multicentre study conducted by Mayer-Hamblett and colleagues involving 269 subjects compiled from 4 different CF studies (1). Despite the limited sample size, the fact we observed similar TL-activity results across two cohorts (one group stable and the other group patients hospitalised for exacerbation) recruited in two separate sites, is reassuring. The different clinical status of the two groups did however limit a full comparison across groups including any substantial investigation of the effect of treatment on airways TLP levels but for cohort 1, a breakdown of patients on therapies such as inhaled or oral antibiotics, inhaled or oral steroid and macrolide was conducted however, no significant differences were observed between the groups (Supplemental Figure S2). All patients in cohort 2 (exacerbation group) were on various mixtures of inhaled, i.v. and oral antibiotics which prevented any further sub-analysis. An understanding of how biomarkers respond to treatment, particularly in the era of triple therapy, is increasingly important. The samples analysed in this study were collected prior to patients having the opportunity to participate even in the early ivacaftor studies. Detailed cell-based studies looking at the mechanisms behind elevated trypsin-like activity levels in $\mathrm{CF}$, to include the effect of CFTR modulation, using differentiated primary human airways epithelial cells grown at air liquid interface is on-going and may further inform the field.

Although widely used, the peptide substrates employed to measure proteolytic activity can be hydrolysed by alternate enzymes present in complex biological samples. To mitigate against this the broad-spectrum TL protease inhibitor (QUB-TL1) was included to improve assay specificity. Consistent with that we find that QUB-TL1, although a broad spectrum inhibitor of TL proteases (9), only partially inhibits the hydrolytic activity against the fluorogenic 
peptide substrate, Boc-QAR-AMC (Supplemental Figure S1A\&B). The profiling of QUB-TL1 against a range of CF lung disease relevant proteases has been included (Supplemental Figure $\mathrm{S} 1 \mathrm{C}$ ), to include the matrix metalloproteases, MMP-2 and -9; the cysteine proteases, cathepsins $\mathrm{B}$ and S; clotting enzymes, Thrombin and Factor $\mathrm{Xa}$, and chymotrypsin and elastase as examples of serine, non-trypsin like proteases. QUB-TL1 selectively targets trypsin-like activity and does not inhibit non-TL serine, cysteine or metalloprotease activity.

QUB-TL1 irreversibly binds the active site of target enzymes (TL proteases) and the incorporation of a biotin tag into its structure enables it to act as an activity-based probe which could facilitate future purification and identification of the specific proteases involved (9). In addition, there may also be opportunity for the development of an activity-based immunoassay to simplify analysis as has been developed for active NE, using a similar ProteaseTag® technology (25). This approach could enable a better understanding of the mechanisms underlying our findings, and may allow a more precise determination of these undoubtedly complex proteases networks in future biomarker and intervention studies.

In conclusion, this exploratory retrospective analysis of adult CF sputum demonstrates for the first time that TLP activity inversely correlates with lung function and patient survival. As such TLP activity warrants further evaluation to determine its usefulness as a biomarker of CF lung disease and/or as a novel therapeutic target. 


\section{Acknowledgements}

JR and KLM were supported by grants to SLM and JSE from the MRC Confidence in Concept (MC_PC_141113) and Cystic Fibrosis Foundation Therapeutics Inc. (MARTIN13XX0), respectively.

\section{Conflict of Interest Statement}

Prof. Martin is a co-founder of ProAxsis Ltd and has received research grants from ProAxsis Ltd. and Cystic Fibrosis Foundation Therapeutics Inc. Prof. Elborn has served as a medical advisor for ProAxsis Ltd, provided consultancy for Bayer and Vertex, and received a grant from Novartis. Dr Reihill, Dr Jones and Dr Moffitt declare no potential conflict of interest.

\section{Author Contributions}

JR and SLM conceived and designed the study. AJ and JSE recruited participants. JR, KM, LED and SLM acquired, analysed and interpreted the data. JR drafted the manuscript which was revised by SLM, JSE, AJ \& KM. All authors approved the final version of the manuscript. 


\section{4. $\quad$ References}

1. Mayer-Hamblett N, Aitken ML, Accurso FJ, Kronmal RA, Konstan MW, Burns JL, et al. Association between pulmonary function and sputum biomarkers in cystic fibrosis. Am $\mathbf{J}$ Respir Crit Care Med. 2007;175(8):822-8.

2. Sagel SD, Wagner BD, Anthony MM, Emmett P, Zemanick ET. Sputum biomarkers of inflammation and lung function decline in children with cystic fibrosis. Am J Respir Crit Care Med. 2012;186(9):857-65.

3. Moffitt KL, Martin SL, Jones AM, Webb AK, Cardwell C, Tunney MM, et al. Inflammatory and immunological biomarkers are not related to survival in adults with Cystic Fibrosis. J Cyst Fibros. 2014;13(1):63-8.

4. Liou TG, Adler FR, Keogh RH, Li Y, Jensen JL, Walsh W, et al. Sputum biomarkers and the prediction of clinical outcomes in patients with cystic fibrosis. PLoS One. 2012;7(8):e42748.

5. Drumm ML, Konstan MW, Schluchter MD, Handler A, Pace R, Zou F, et al. Genetic modifiers of lung disease in cystic fibrosis. N Engl J Med. 2005;353(14):1443-53.

6. Twigg MS, Brockbank S, Lowry P, FitzGerald SP, Taggart C, Weldon S. The Role of Serine Proteases and Antiproteases in the Cystic Fibrosis Lung. Mediators Inflamm. 2015;2015:293053.

7. McKelvey MC, Weldon S, McAuley DF, Mall MA, Taggart CC. Targeting Proteases in Cystic Fibrosis Lung Disease: Paradigms, Progress, and Potential. Am J Respir Crit Care Med. 2019.

8. Martin SL, Moffitt KL, McDowell A, Greenan C, Bright-Thomas RJ, Jones AM, et al. Association of airway cathepsin B and S with inflammation in cystic fibrosis. Pediatr Pulmonol. 2010;45(9):860-8.

9. Reihill JA, Walker B, Hamilton RA, Ferguson TE, Elborn JS, Stutts MJ, et al. Inhibition of Protease-Epithelial Sodium Channel Signaling Improves Mucociliary Function in Cystic Fibrosis Airways. Am J Respir Crit Care Med. 2016;194(6):701-10.

10. McMahon DB, Workman AD, Kohanski MA, Carey RM, Freund JR, Hariri BM, et al. Protease-activated receptor 2 activates airway apical membrane chloride permeability and increases ciliary beating. FASEB J. 2018;32(1):155-67.

11. Moore PJ, Tarran R. The epithelial sodium channel $(\mathrm{ENaC})$ as a therapeutic target for cystic fibrosis lung disease. Expert Opin Ther Targets. 2018;22(8):687-701. 
12. Martin SL, Saint-Criq V, Hwang TC, Csanady L. Ion channels as targets to treat cystic fibrosis lung disease. J Cyst Fibros. 2018;17(2S):S22-S7.

13. Shei RJ, Peabody JE, Kaza N, Rowe SM. The epithelial sodium channel (ENaC) as a therapeutic target for cystic fibrosis. Curr Opin Pharmacol. 2018;43:152-65.

14. Kleyman TR, Carattino MD, Hughey RP. ENaC at the cutting edge: regulation of epithelial sodium channels by proteases. J Biol Chem. 2009;284(31):20447-51.

15. Myerburg MM, McKenna EE, Luke CJ, Frizzell RA, Kleyman TR, Pilewski JM. Prostasin expression is regulated by airway surface liquid volume and is increased in cystic fibrosis. Am J Physiol Lung Cell Mol Physiol. 2008;294(5):L932-41.

16. Nimishakavi S, Besprozvannaya M, Raymond WW, Craik CS, Gruenert DC, Caughey GH. Activity and inhibition of prostasin and matriptase on apical and basolateral surfaces of human airway epithelial cells. Am J Physiol Lung Cell Mol Physiol. 2012;303(2):L97-106.

17. Boucher RC. Evidence for airway surface dehydration as the initiating event in $\mathrm{CF}$ airway disease. J Intern Med. 2007;261(1):5-16.

18. Heuberger DM, Schuepbach RA. Protease-activated receptors (PARs): mechanisms of action and potential therapeutic modulators in PAR-driven inflammatory diseases. Thromb J. 2019; $17: 4$.

19. Ossovskaya VS, Bunnett NW. Protease-activated receptors: contribution to physiology and disease. Physiol Rev. 2004;84(2):579-621.

20. Dulon S, Cande C, Bunnett NW, Hollenberg MD, Chignard M, Pidard D. Proteinaseactivated receptor-2 and human lung epithelial cells: disarming by neutrophil serine proteinases. Am J Respir Cell Mol Biol. 2003;28(3):339-46.

21. Chokki M, Yamamura S, Eguchi H, Masegi T, Horiuchi H, Tanabe H, et al. Human airway trypsin-like protease increases mucin gene expression in airway epithelial cells. Am J Respir Cell Mol Biol. 2004;30(4):470-8.

22. Liu C, Li Q, Zhou X, Kolosov VP, Perelman JM. Human airway trypsin-like protease induces mucin5 AC hypersecretion via a protease-activated receptor 2-mediated pathway in human airway epithelial cells. Arch Biochem Biophys. 2013;535(2):234-40.

23. Laporte M, Naesens L. Airway proteases: an emerging drug target for influenza and other respiratory virus infections. Curr Opin Virol. 2017;24:16-24.

24. Jones AM, Martin L, Bright-Thomas RJ, Dodd ME, McDowell A, Moffitt KL, et al. Inflammatory markers in cystic fibrosis patients with transmissible Pseudomonas aeruginosa. Eur Respir J. 2003;22(3):503-6. 
25. Chalmers JD, Moffitt KL, Suarez-Cuartin G, Sibila O, Finch S, Furrie E, et al. Neutrophil Elastase Activity Is Associated with Exacerbations and Lung Function Decline in Bronchiectasis. Am J Respir Crit Care Med. 2017;195(10):1384-93.

26. Caldwell RA, Boucher RC, Stutts MJ. Neutrophil elastase activates near-silent epithelial $\mathrm{Na}+$ channels and increases airway epithelial $\mathrm{Na}+$ transport. Am J Physiol Lung Cell Mol Physiol. 2005;288(5):L813-9.

27. Belaaouaj A, McCarthy R, Baumann M, Gao Z, Ley TJ, Abraham SN, et al. Mice lacking neutrophil elastase reveal impaired host defense against gram negative bacterial sepsis. Nat Med. 1998;4(5):615-8.

28. Hirche TO, Benabid R, Deslee G, Gangloff S, Achilefu S, Guenounou M, et al. Neutrophil elastase mediates innate host protection against Pseudomonas aeruginosa. J Immunol. 2008;181(7):4945-54.

29. Gehrig S, Duerr J, Weitnauer M, Wagner CJ, Graeber SY, Schatterny J, et al. Lack of neutrophil elastase reduces inflammation, mucus hypersecretion, and emphysema, but not mucus obstruction, in mice with cystic fibrosis-like lung disease. Am J Respir Crit Care Med. 2014;189(9):1082-92.

30. Wang J, Zhu HH, Xue JH, Wu SS, Chen Z. Effects of storage conditions on the stability of serum CD163, NGAL, HMGB1 and MIP2. Int J Clin Exp Pathol. 2015;8(4):4099105. 